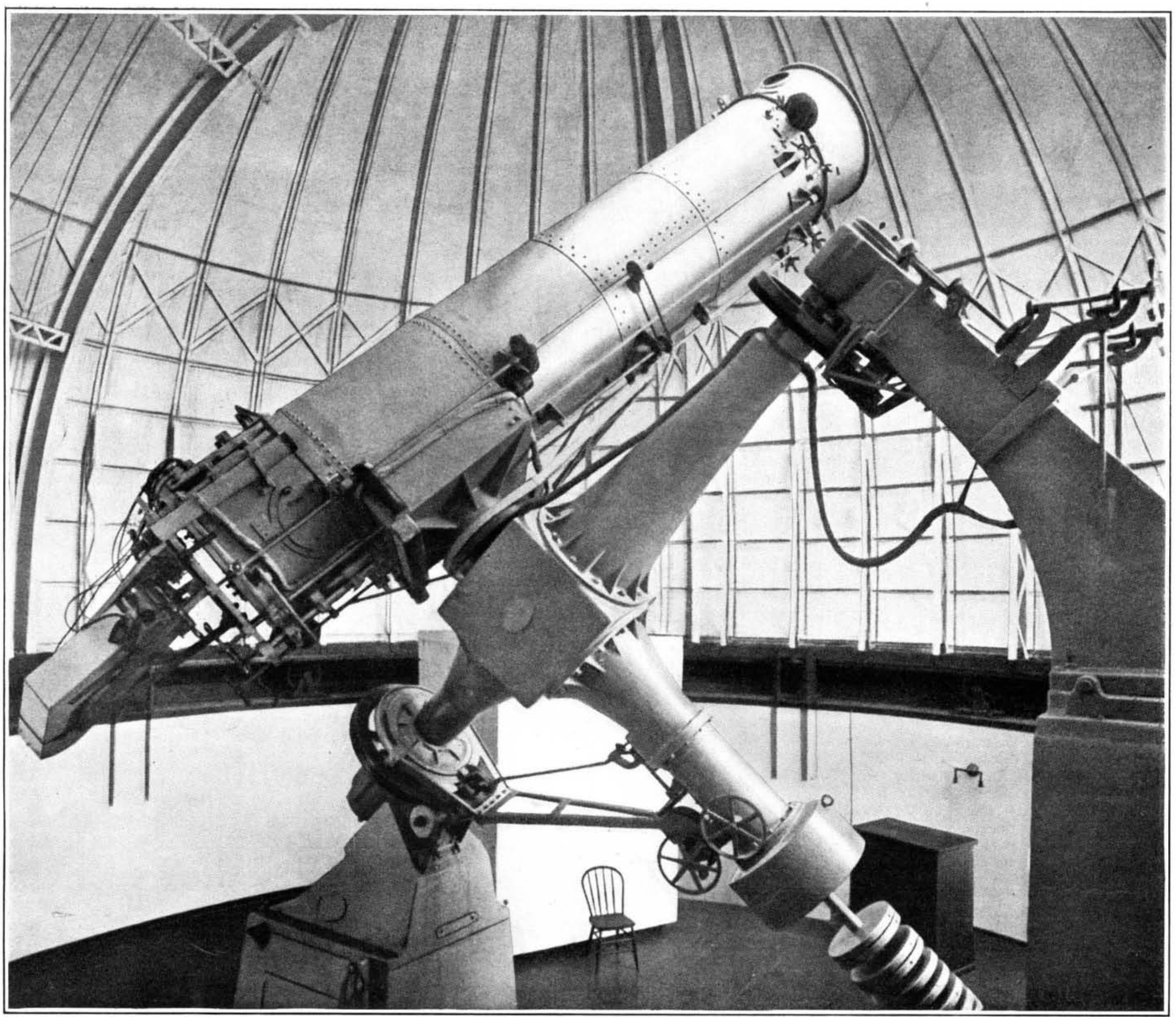

Fig. 1. The thirty-seven and one-half inch reflecting telescope of the Observatory of the University of Michigan

\title{
Stellar Evolution
}

The Discovery of a Missing Link in the Chain by Spectra Observations

R. H. Curtiss, Associate Professor of Astronomy, University of Michigan

ONE of the first generalizations drawn from extensive observations of stellar spectra was the recognition of a sequence in which there was a place for nearly every star examined. In the interest of convenience certain definitely characterized types in this sequence were chosen as a frame work for classification first by Secchi, later by Vogel, and by Lockyer, and lastly, on the most complete basis by investigators at the Harvard College Observatory.

The Harvard Classification of Stellar Spectra, using the letters $\mathrm{O}, \mathrm{B}, \mathrm{A}, \mathrm{F}, \mathrm{G}, \mathrm{K}$ and $\mathrm{M}$ to designate the divisions, is adopted almost universally in this country, and is widely used in Europe. On the one end are placed white or bluish-white stars whose spectra show well defined nebular characteristics and on the other mell defined nebular bands of compounds as well as by many of the metallic absorption lines found in the solar spectrum, which in the Harvard Classification is assigned to Class G. Practically all astronomers agree in the view that the sequence of letters in the Harvard Classification corresponds to stages of stellar development from the white hot, low density star on the one hand to the relatively cool red star on the other approaching a state of visual extinction, and that the divisions in the spectral sequence of the Harvard Classification serve as an index to successive physical states in the process of stellar evolution.
Accordingly, an interruption in this sequence may be regarded as a missing link in the chain of stellar evolution. Corresponding to a wider range of observation and increased knowledge the Harvard system of classification has been slowly extended to include more and more objects for some of which no previous provision had been made, so that now there remain outstanding only a few isolated cases or small groups of stellar bodies whose relation to the regular sequence is not clear.

Occupying a prominent position among outstanding groups of intractable objects are those stars whose spectra have been placed in a division called Class $\mathbf{N}$ by the Harvard investigators. In a recent Harvard tabulation 267 of these objects are found. They are all red stars. Among them no bright members are found. All of them are probably very distant and a large majority of them are located in the Milky Way. The spectra of these stars are very similar to those of Class $M$ at the end of the sequence given above. The chief difference between spectra of Class $\mathbf{M}$ and Class $\mathbf{N}$ lie in the special absorption. Class $M$ spectra contai wide absorption bands sharp toward the violet and degraded toward the red, probably due to titanium oxide; Class $\mathbf{N}$ spectra have similar bands, though reversed in appearance, being sharp toward the red, attributed to some carbon compound.

Since these carbon and titanium oxide absorptio bands are usually conspicuous features in these spectra many investigators beginning with the earliest have held the view that the spectra of Class $M$ and Class $\mathbf{N}$ should all into two distinectra of Class $M$ and Class $N$ Class $M$ appears to be attached quite directly to the spectral equence Other investigators have looked past the bolder features of the bands and have concluded from the resemblance in the remaining characteristics that these two types of spectra should be classed together as coördinate groups leading back to the sun.

Probably most astronomers have accepted this latter conclusion. But a serious objection to the proposal of coördinate branches leading back to the sun has been based on the fact that no examples of an intermediate type between the solar spectrum and Class $\mathbf{N}$ had been found though a complete sequence from the sun to Class $M$ had long been established. There was evidence of a missing link in the proposed chain of stellar evolution connecting stars with spectra like that of the sun and those with spectra of Class $\mathbf{N}$.

Not long before this time several unusual stars had been swept up in the course of the extensive spectral surveys which were under way at the Harvard College Observatory. These objects though placed in Class N, were found to be less red or more like the sun in color, than ordinary objects of this class. Later discoveries of stars with spectra of this kind increased the number 
to the point where the addition of a new class was warranted. Accordingly Class $R$ was created to include them. Because of the faintness of the Class $R$ stars spectroscopists met with great difficulties in study ing their spectra but there was evidence sufficient to suggest that the stars of Class $\mathrm{R}$ might supply the missing link between the sun and Class $\mathbf{N}$ stars.

At this juncture the new $371 / 2$-inch reflector (see Fig. 1) of the Observatory of the University of Michigan became available for research with its great light-gathering power. This instrument had been fitted with a slit power. This in reume spectrograph well ten of the brightest of the Class $R$ stars not too far south of the equator was undertaken at this Observatory. The investigation was assigned to $\mathrm{Mr}$. W. Carl Rufus who developed the study into a thesis accepted in partia fulfillment of the requirements for the degree of doctor of philosophy in the University of Michigan, and published in Volume II of the Publications of the Observatory of the University of Michigan.

The results of this study of the spectra of Class $R$ stars can be set forth best in a non-technical journa through the accompanying illustration. (See Fig. 2). In this the spectrum of our sun is placed at the top and a standard Class $\mathbf{N}$ stellar spectrum at the bottom. tween these are ranged in a sequential order eight Class $\mathbf{R}$ and Class $\mathbf{N}$ stellar spectra showing successive stcps in the transition from the solar spectrum to that of a standard Class $\mathbf{N}$ star. The first of these Class $R$ spectra is quite similar to that of the sun immediately above it in the illustration. The chief difference between these two spectra consists first in the relatively greater faintness of the Class $R$ spectrum on the left or violet end, due both to increased general absorption and to special absorption of cyanogen beginning under the letter " $g$," and secondly in the presence of the characteristic "carbon absorption band" in the Class R spectrum about one-third of the distance from the right or green end to the left or violet end. A little lower down in the series absorption due to carbon appears beginning almost directly under the symbol " 24405 ." These three bold features become more prominent as we proceed down the sequence until in the standard Class $\mathbf{N}$ spectrum at the bottom we have a short band of ligh in this region divided by a heavy black carbon band On the basis of the series of spectra shown here, and of others photographed at Ann Arbor, the fact seem well established that the spectra of Class $R$ supply the missing steps between the spectra of Class $\mathbf{N}$ and the ming port is given to the theory that the two classes of red stars, Classes $M$ and $N$, are coördinate groups leading back to the sun. If this convincing evidence be accepted, the relation of Class $\mathbf{N}$ through Class $\mathbf{R}$ to the spectral sequence is defimed; a gap is closed in the order of stellar spectral classes; and in the Class $\mathrm{R}$ stars is found

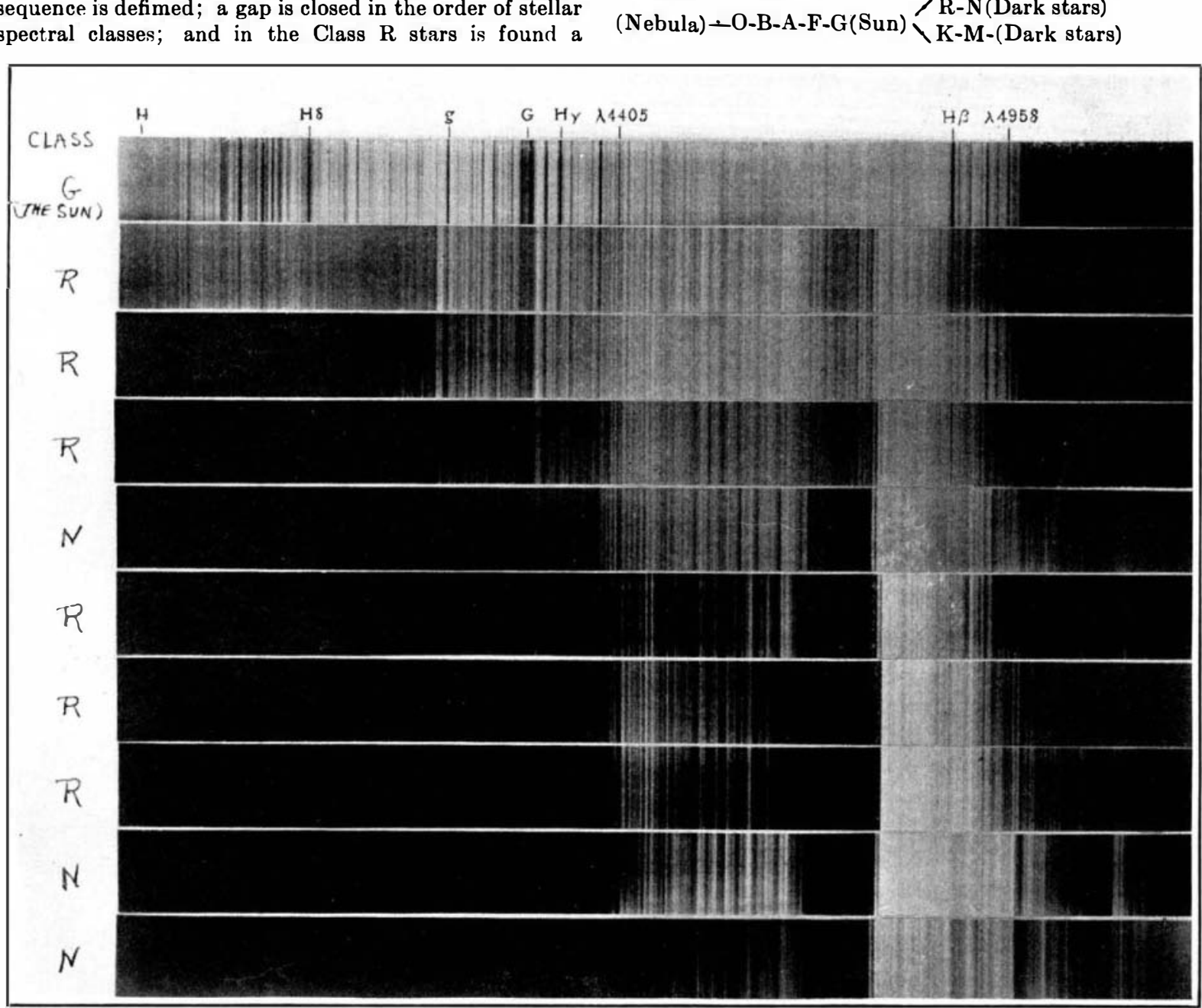

Fig. 2. Stellar spectra, showing steps in evolutionary sequence from the Sun (Class $\left.{ }^{7} G\right)$ to the standard Class $\mathbf{N}$ star insing link in the evolutionary chain which is thought to be formed by the successive stages in the life of a of the letters denoting the divisions of the Harvard Classification of stellar spectra may be indicated by the following arrangement of symbols with the new link, Class $\mathbf{R}$, introduced:

Nebula)-O-B-A-F-G(Sun) $/$ R-N(Dark stars)

\title{
Maintenance of Management
}

\section{The Most Important Element of Waste Elimination}

\author{
By Frank B. Gilbreth, Mem. Am. Soc. M. E.
}

A MOsT important element of waste elimination is maintenance of the best that is known. Achievement in the successful installation of new features of management depends more upon maintenance of the best than it does upon WHAT has been installed and HOW the component parts have been introduced or WHO has been taught. The work of changing management, or of installing new management, demands five things:

1. Knowledge of the underlying laws of management.

2. Procedure for determining methods of least waste. 3. The least wasteful sequence of installation of features of Modern Management.

4. Procedure for teaching the methods of least aste.

5. Maintenance of standards.

Too of ten there is a tendency to consider that a successful demonstration is all that is needed to prove the completeness of the efficiency of the new method. In reality, unless the desired features are maintained permanently, there is little likelihood of a return of benefits sufficien to pay the cost of installation. The purpose of this pape is to show a method that will assist in handling the problem of maintenance, by describing in detail the making and the use of the "Standing Order"

There are various requirements that a Standing Order must fulfill if it is to act as a maintaining force. (1) The first requirement in the process of maintenance is a clear understanding in the mind of the management as to WHAT we wish to maintain. The best way to insure and to perpetuate this is to put it in writing. The subject matter of the standing order may then be considered and used as "of the essence of the contract" between the employer and the employee, in determining whether or not the high rate to be paid only upon the fulfillment of the terms of the Standing Order, has been earned.

2. The second requirement of a Standing Order is recognition of WHY the thing required is to be done. In the course of time, the file of Standing Orders naturally becomes voluminous; consequently, no one can be expected to remember them all. In fact, in many cases, it will be difficult to remember even all of those that affect one's own line of promotion. Yet, the more that are remembered by all, the better will be the result, We have found during a continuous experience of 20 years of using written Standing Orders, that the more the WHY is inserted in the Standing Order, the easier it is to remember it. Furthermore, it is more natural for one to coöperate heartily toward the desired end when one sees the reason, the purpose and the underlying motive of the Standing Order.

3. The third requirement is a clear statement as to WHO is responsible for the order. Oftentimes the subject matter of a Standing Order is perfectly clear in the mind of the one who suggested it, but it is not clear as written on the Standing Order form. Therefore, what is in effect a Standing Orders Committee passes upo each Standing Order. This committee consists of:

a. The suggester of the idea in its original form (The original form may be quite different from it effective, or final, form.)

b. The suggester of any previous Standing Order that it affects.

c. The approver of all Standing Orders. This ma should be one who has sufficient grasp on the subject matter of all Standing Orders to understand the effect of the additions or changes that the new Standing Orde will have.

d. The decider who makes the Standing Order, as written, effective or void. He should be the best man obtainable, one who is willing to have his measure take by his decisions and to take the glory or blame of his decisions.

The committee is supplemented in the followin manner: Many suggestions for new Standing Orders 